\title{
Continued Fractions of Different Quotients
}

\author{
Roselin Antony \\ Dept. of Mathematics, College of Natural and Computational Sciences, Mekelle University, Mekelle, Ethiopia \\ *Corresponding Author: Roselin Antony, Dept. of Mathematics, College of Natural and Computational \\ Sciences, Mekelle University, Mekelle, Ethiopia
}

\begin{abstract}
The use of continued fractions as an important tool in number theory began with $17^{\text {th }}$ century results of Schwenter, Huygens and Wallis and came to maturity with the work of Euler in 1737 and the subsequent use of continued fractions as a number theoretic tool by Lagrange, Legendre, Gauss, Galois and their successors. In this paper, we will find the continued fraction representation of quotients of different powers of consecutive numbers between 2 and 10.
\end{abstract}

Keywords: Continued fractions, quotients, rational approximations, Euclidean algorithm.

\section{INTRODUCTION}

The efficient process of finding the best rational approximations of any real number $x$ is the continued fraction expansion of that number. In general, a simple continued fraction is an expression of the form

$$
\left[a_{0}, a_{1}, \ldots . . .\right]=a_{0}+\frac{1}{a_{1}+\frac{1}{a_{2}+\ldots . .}} .
$$

where the letters $a_{0}, a_{1}, a_{2}, \ldots .$. denote independent variables and may be interpreted as real or complex numbers, functions etc. If the number of terms is finite, we write

$$
\begin{array}{r}
{\left[a_{0}, a_{1}, \ldots \ldots a_{n}\right]=a_{0}+\frac{1}{a_{1}+\frac{1}{a_{2}+\ldots .}}} \\
+\frac{1}{a_{n}}
\end{array}
$$

If the number of terms is infinite, we write

$$
\left[a_{0}, a_{1}, \ldots . . .\right]=a_{0}+\frac{1}{a_{1}+\frac{1}{a_{2}+\ldots . .}}
$$

For a finite continued fraction $\left[a_{0}, a_{1}, a_{2}, \ldots . ., a_{n}\right]$ and a positive integer $k \leq n$, the k-th remainder is defined as the continued fraction

$$
r_{k}=\left[a_{k}, a_{k+1}, \ldots . ., a_{n}\right] \text {. }
$$

Any rational number can be represented as a finite continued fraction. If $x=a / b$ is a rational number, then the method for obtaining the continued fraction of $x$ is nothing else than the Euclidean algorithm for computing the greatest common divisor of $a$ and $b$ :

$$
\begin{array}{lll}
a=a_{0} b+r_{0}, & 0 \leq r_{0} \leq b, & x_{1}=b / r_{0}, \\
b=a_{1} r_{0}+r_{1}, & 0 \leq r_{1} \leq r_{0}, & x_{2}=r_{0} / r_{1}, \\
r_{0}=a_{2} r_{1}+r_{2}, & 0 \leq r_{2} \leq r_{1}, & x_{3}=r_{1} / r_{2},
\end{array}
$$


Therefore, on the other hand, since the Euclidean algorithm always stops, the continued fraction of a rational number is always finite. Hence, it is obvious that a finite continued fraction represents a rational number[1].

In view of unwieldiness of this notation, various authors have proposed other ways of writing continued fractions, for example

$$
\begin{aligned}
& b_{0}+\frac{a_{1} \mid}{\mid b_{1}}+\frac{a_{2} \mid}{\mid b_{2}}+\ldots . .+\frac{a_{n} \mid}{\mid b_{n}} \\
& b_{0}+\frac{a_{1}}{b_{1}}+\frac{a_{2}}{b_{2}}+\ldots . .+\frac{a_{n}}{b_{n}}+\ldots \ldots . \\
& b_{0}+\frac{a_{1}}{b_{1}}+\frac{a_{2}}{b_{2}}+\ldots . .+\frac{a_{n}}{b_{n}}+\ldots \ldots .
\end{aligned}
$$

where the fraction $\frac{a_{n}}{b_{n}}$ is called the $\mathrm{n}^{\text {th }}$ partial quotient of the continued fraction; $\mathrm{a}_{\mathrm{n}}$ and $\mathrm{b}_{\mathrm{n}}$ are the coefficients of the continued fraction; $b_{1}, b_{2}, \ldots .$. its partial denominators; $a_{1}, a_{2} \ldots .$. its partial numerators.

The study of finite continued fractions, began in its explicit form in the latter decades of the $16^{\text {th }}$ century with a paper by Bombelli written when the concepts and notations of algebra were first being laid down in Italy and France such expressions play a natural role in connection with the integrated application of the Euclidean algorithm and some mathematical historians have claimed to have found similar usages in Hindu or even Greek mathematics. Infinite continued fractions were first considered by Lord Broucker, first president of the Royal Society.

The use of continued fractions as an important tool in number theory began with $17^{\text {th }}$ century results of Schwenter, Huygens and Wallis and came to maturity with the work of Euler in 1737 and the subsequent use of continued fractions as a number theoretic tool by Lagrange, Legendre, Gauss, Galois and their successors. Continued fraction expansions involving functions of a complex variable rather than simply numbers were introduced by Euler and became an important tool in the approximation of special classes of analytic functions in the work of Euler, Lambert and Lagrange.

Ramanujan's continued fraction of order 5 is given by,

$$
R_{1}(q)=1+\frac{q}{1+} \frac{q^{2}}{1+} \frac{q^{3}}{1+\ldots \ldots \ldots . . .}
$$

Ramanujan's continued fraction of order 6 is

$$
R(q)=1+\frac{q(1+q)}{1+} \frac{q^{2}\left(1+q^{2}\right)}{1+} \frac{q^{3}\left(1+q^{3}\right)}{1+} \ldots \ldots \ldots . . .
$$

Ramanujan [5] gave the following result

$$
1+\frac{q(1+q)}{1+} \frac{q^{2}\left(1+q^{2}\right)}{1+} \frac{q^{3}\left(1+q^{3}\right)}{1+} \ldots \ldots \ldots=\frac{\left(q^{3}, q^{3} ; q^{6}\right)_{\infty}}{\left(q, q^{5} ; q^{6}\right)_{\infty}}
$$

Andrews [6] gave the following continued fraction

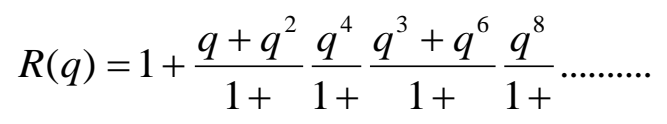




\section{MAIN RESULTS}

Let us consider the natural numbers from 2 to 10. In this paper, we will find the continued fraction representation of quotients of different powers of consecutive numbers between 2 and 10.

\subsection{Quotients of 3 and 2 with powers of 2 to 10}

Let $A_{1}, A_{2}, A_{3}, \ldots . ., A_{9}$ represent the quotients of the numbers 3 and 2 raised to the same powers starting from power 2 to power 10 .

$$
\begin{aligned}
& A_{1}=2+\frac{1}{4}=[2,4] \text {. } \\
& A_{2}=3+\frac{1}{2+\frac{1}{1+\frac{1}{2}}}=[3,2,1,2] \text {. } \\
& A_{3}=5+\frac{1}{16}=[5,16] \text {. } \\
& A_{4}=7+\frac{1}{1+\frac{1}{1+\frac{1}{4+\frac{1}{3}}}}=[7,1,1,4,3] \text {. } \\
& A_{5}=11+\frac{1}{2+\frac{1}{1+\frac{1}{1+\frac{1}{3+\frac{1}{1+\frac{1}{2}}}}}}=[11,2,1,1,3,1,2] \text {. } \\
& A_{6}=17+\frac{1}{11+\frac{1}{1+\frac{1}{1+\frac{1}{1+\frac{1}{3}}}}}=[17,11,1,1,1,3] \text {. } \\
& A_{7}=25+\frac{1}{1+\ldots . .}=[25,1,1,1,2,3,1,1,1,2] \text {. } \\
& 1+\frac{1}{1+\frac{1}{2}} \\
& A_{8}=38+\frac{1}{2+\ldots \ldots .}=[38,2,3,1,10,1,1,2] \text {. } \\
& 1+\frac{1}{1+\frac{1}{2}}
\end{aligned}
$$




$$
\begin{aligned}
& A_{9}=57+\frac{1}{1+\ldots \ldots}=[57,1,1,1,67,1,1,2] . \\
& 1+\frac{1}{1+\frac{1}{2}}
\end{aligned}
$$

\subsection{Quotients of 4 and 3 with powers of 2 to 10}

Let $\mathrm{B}_{1}, \mathrm{~B}_{2}, \mathrm{~B}_{3}, \ldots . ., \mathrm{B}_{9}$ represent the quotients of the numbers 4 and 3 raised to the same powers starting from power 2 to power 10.

$$
\begin{aligned}
& B_{1}=1+\frac{1}{1+\frac{1}{3+\frac{1}{2}}}=[1,1,3,2] \text {. } \\
& B_{2}=2+\frac{1}{2+\frac{1}{1+\frac{1}{2+\frac{1}{3}}}}=[2,2,1,2,3] \text {. } \\
& B_{3}=3+\frac{1}{6+\frac{1}{4+\frac{1}{3}}}=[3,6,4,3] \text {. } \\
& B_{4}=4+\frac{1}{4+\frac{1}{1+\frac{1}{2+\frac{1}{17}}}}=[4,4,1,2,17] \text {. } \\
& B_{5}=5+\frac{1}{1+\ldots \ldots}=[5,1,1,1,1,1,1,1,5,6] . \\
& 1+\frac{1}{5+\frac{1}{6}} \\
& B_{6}=7+\frac{1}{2+\frac{1}{29+\frac{1}{18+\frac{1}{2}}}}=[7,2,29,18,2] \text {. } \\
& B_{7}=9+\frac{1}{1+\frac{1}{87+\frac{1}{1+\frac{1}{1+\frac{1}{1+\frac{1}{24}}}}}}=[9,1,87,1,1,1,24] \text {. }
\end{aligned}
$$




$$
\begin{gathered}
B_{8}=13+\frac{1}{3+\frac{1}{7+\frac{1}{18+\frac{1}{8+\frac{1}{6}}}}}=[13,3,7,18,8,6] . \\
B_{9}=17+\frac{1}{1+\ldots . .}=[17,1,3,7,1,5,4,1,4,1,1,5] . \\
1+\frac{1}{1+\frac{1}{5}}
\end{gathered}
$$

\subsection{Quotients of 5 and 4 with powers of 2 to 10}

Let $\mathrm{C}_{1}, \mathrm{C}_{2}, \mathrm{C}_{3}, \ldots ., \mathrm{C}_{9}$ represent the quotients of the numbers 5 and 4 raised to the same powers starting from power 2 to power 10 .

$$
\begin{aligned}
& C_{1}=1+\frac{1}{1+\frac{1}{1+\frac{1}{3+\frac{1}{2}}}}=[1,1,1,3,2] . \\
& C_{2}=1+\frac{1}{1+\frac{1}{20+\frac{1}{3}}}=[1,1,20,3] . \\
& C_{3}=2+\frac{1}{2+\ldots \ldots .}=[2,2,2,1,6,1,1,2] . \\
& C_{4}=3+\frac{1+\frac{1}{1+\frac{1}{2}}}{19+\frac{1}{3+\frac{1}{8+\frac{1}{2}}}}=[3,19,3,8,2] . \\
& C_{6}=4+\frac{1}{1+\ldots . .} \\
& C_{5}=3+\frac{1}{1+\ldots \ldots . .}
\end{aligned}
$$




$$
\begin{aligned}
& C_{7}=5+\frac{1}{1+\ldots . .} \\
& 2+\frac{1}{8+\frac{1}{18}} \\
& C_{8}=7+\frac{1}{2+\ldots . .}=[5,1,24,3,2,2,8,18] . \\
& 7+\frac{1}{1+\frac{1}{2}} \\
& C_{9}=9+\frac{1}{3+\ldots . .} \quad 1+\frac{1}{1+\frac{1}{2}}
\end{aligned}
$$

\subsection{Quotients of 6 and 5 with powers of 2 to 10}

Let $\mathrm{D}_{1}, \mathrm{D}_{2}, \mathrm{D}_{3}, \ldots . ., \mathrm{D}_{9}$ represent the quotients of the numbers 6 and 5 raised to the same powers starting from power 2 to power 10 .

$$
\begin{aligned}
& D_{1}=1+\frac{1}{2+\frac{1}{3+\frac{1}{1+\frac{1}{2}}}=}[1,2,3,1,2] . \\
& D_{2}=1+\frac{1}{1+\frac{1}{2+\frac{1}{1+\frac{1}{2+\frac{1}{11}}}}}=[1,1,2,1,2,11] . \\
& D_{3}=2+\frac{1}{13+\ldots . .} \\
& D_{4}=2+\frac{1+\frac{1}{2+\ldots . .}}{9+\frac{1}{2+\frac{1}{3}}} \\
& D_{5}=3+\frac{1}{187+\frac{1}{20+\frac{1}{1+\frac{1}{3}}}}
\end{aligned}
$$




$$
\begin{aligned}
& D_{6}=3+\frac{1}{1+\ldots . .}=[3,1,1,2,1,1,44,1,16,1,7] . \\
& 6+\frac{1}{1+\frac{1}{7}} \\
& D_{7}=4+\frac{1}{3+\ldots . .}=[4,3,2,1,53,1,13,1,8,1,1,2] . \\
& D_{8}=5+\frac{1+\frac{1}{6+\ldots . .}}{1+\frac{1}{2}}=[5,6,3,1,6,1,1,9,1,3,18,2,3] . \\
& D_{9}=6+\frac{1}{5+\ldots . .} \quad \frac{1}{3+\frac{1}{2+\frac{1}{3}}}
\end{aligned}
$$

\subsection{Quotients of 7 and 6 with powers of 2 to 10}

Let $E_{1}, E_{2}, E_{3}, \ldots . ., E_{9}$ represent the quotients of the numbers 7 and 6 raised to the same powers starting from power 2 to power 10 .

$$
\begin{aligned}
& E_{1}=1+\frac{1}{2+\frac{1}{1+\frac{1}{3+\frac{1}{3}}}}=[1,2,1,3,3] . \\
& E_{2}=1+\frac{1}{1+\ldots \ldots .}=[1,1,1,2,2,1,12] . \\
& E_{3}=1+\frac{1}{5+\ldots \ldots . \frac{1}{12}} \\
& \quad 1+\frac{1}{1+\frac{1}{13}} \\
& E_{4}=2+\frac{1}{6+\ldots \ldots .} \\
& 5+\frac{1}{1+\frac{1}{36}}
\end{aligned}
$$




$$
\begin{aligned}
& E_{5}=2+\frac{1}{1+\ldots \ldots}=[2,1,1,11,184,2,1,3] . \\
& 2+\frac{1}{1+\frac{1}{3}} \\
& E_{6}=2+\frac{1}{1+\ldots . .}=[2,1,16,4,1,2,1,5,1,8,1,12] . \\
& 8+\frac{1}{1+\frac{1}{12}} \\
& E_{7}=3+\frac{1}{2+\ldots \ldots}=[3,2,3,5,3,6,1,2,26,1,5,4] . \\
& 1+\frac{1}{5+\frac{1}{4}} \\
& E_{8}=4+\frac{1}{235+\ldots . .}=[4,235,2,1,284,1,4,1,1,4] . \\
& 1+\frac{1}{1+\frac{1}{4}} \\
& E_{9}=4+\frac{1}{1+\ldots \ldots}=[4,1,2,22,12,1,1,2,6,2,2,83,1,1,2] . \\
& 1+\frac{1}{1+\frac{1}{2}}
\end{aligned}
$$

\subsection{Quotients of 8 and 7 with powers of 2 to 10}

Let $F_{1}, F_{2}, F_{3}, \ldots . ., F_{9}$ represent the quotients of the numbers 8 and 7 raised to the same powers starting from power 2 to power 10 .

$$
\begin{aligned}
& F_{1}=1+\frac{1}{3+\frac{1}{3+\frac{1}{1+\frac{1}{3}}}}=[1,3,3,1,3] . \\
& F_{2}=1+\frac{1}{2+\frac{1}{33+\frac{1}{1+\frac{1}{4}}}}=[1,2,33,1,4] . \\
& F_{3}=1+\frac{1}{1+\frac{1}{2+\frac{1}{3+\frac{1}{6+\frac{1}{26}}}}}
\end{aligned}
$$




$$
\begin{aligned}
& F_{4}=1+\frac{1}{1+\ldots \ldots}=[1,1,18,1,6,2,18,3] . \\
& 2+\frac{1}{18+\frac{1}{3}} \\
& F_{5}=2+\frac{1}{4+\ldots \ldots}=[2,4,2,1,1,1,1,2,65,2,2,2] . \\
& 2+\frac{1}{2+\frac{1}{2}} \\
& F_{6}=2+\frac{1}{1+\ldots \ldots}=[2,1,1,4,1,7,11,4,1,7,1,1,1,6] . \\
& 1+\frac{1}{1+\frac{1}{6}} \\
& F_{7}=2+\frac{1}{1+\ldots . .}=[2,1,10,6,1,4,1,4,2,6,1,16,1,3,2] . \\
& 1+\frac{1}{3+\frac{1}{2}} \\
& F_{8}=3+\frac{1}{3+\ldots \ldots}=[3,3,14,1,9,4,1,1,7,20,31,2] . \\
& 20+\frac{1}{31+\frac{1}{2}} \\
& F_{9}=3+\frac{1}{1+\ldots \ldots}=[3,1,4,33,2,3,1,2,6,3,2,2,4,6,2,4,2] \text {. } \\
& 2+\frac{1}{4+\frac{1}{2}}
\end{aligned}
$$

\subsection{Quotients of 9 and 8 with powers of 2 to 10}

Let $G_{1}, G_{2}, G_{3}, \ldots . ., G_{9}$ represent the quotients of the numbers 9 and 8 raised to the same powers starting from power 2 to power 10 .

$$
\begin{gathered}
G_{1}=1+\frac{1}{3+\frac{1}{1+\frac{1}{3+\frac{1}{4}}}}=[1,3,1,3,4] . \\
G_{2}=1+\frac{1}{2+\ldots \ldots .}=[1,2,2,1,3,1,1,2,3] . \\
1+\frac{1}{2+\frac{1}{3}}
\end{gathered}
$$




$$
\begin{gathered}
G_{3}=1+\frac{1}{2+\ldots \ldots .} \\
8+\frac{1}{1+\frac{1}{29}}
\end{gathered}=[1,2,1,3,1,8,1,29] .
$$

$$
G_{4}=1+\frac{1}{1+\ldots \ldots}=[1,1,4,19,2,12,3,4] .
$$

$$
\begin{aligned}
& 12+\frac{1}{3+\frac{1}{4}} \\
& G_{5}=2+\frac{1}{36+\ldots . .}=[2,36,1,1,1,5,3,11,1,2,1,2] . \\
& 2+\frac{1}{1+\frac{1}{2}}
\end{aligned}
$$

$$
G_{6}=2+\frac{1}{3+\ldots \ldots}=[2,3,1,1,3,2,69,2,1,1,1,21,3] .
$$

$$
1+\frac{1}{21+\frac{1}{3}}
$$

$$
G_{7}=2+\frac{1}{1+\ldots \ldots}=[2,1,1,3,3,3,38,3,6,1,2,3,3,1,1,3] .
$$

$$
1+\frac{1}{1+\frac{1}{3}}
$$

$$
G_{8}=2+\frac{1}{1+\ldots \ldots}=[2,1,7,1,4,3,2,1,1,1,1,7,1,3,1,1,5,1,9,3,4] .
$$

$$
9+\frac{1}{3+\frac{1}{4}}
$$

$$
\begin{aligned}
& G_{9}=3+\frac{1}{4+\ldots \ldots .} \\
& 2+\frac{1}{8+\frac{1}{6}}
\end{aligned}=[3,4,23,12,1,1,8,2,20,1,10,2,8,6] .
$$




$$
\begin{gathered}
H_{1}=1+\frac{1}{4+\frac{1}{3+\frac{1}{1+\frac{1}{4}}}}=[1,4,3,1,4] . \\
H_{2}=1+\frac{1}{2+\ldots . .}=[1,2,1,2,4,2,2,1,2] \\
2+\frac{1}{1+\frac{1}{2}}
\end{gathered}
$$

$H_{3}=1+\frac{1}{1+\ldots \ldots}=[1,1,1,9,1,5,1,1,1,1,9]$

$$
1+\frac{1}{1+\frac{1}{9}}
$$

$H_{4}=1+\frac{1}{1+\ldots \ldots}=[1,1,2,1,4,9,1,4,3,1,1,2]$

$$
H_{5}=1+\frac{1+\frac{1}{1+\frac{1}{2}}}{1+\ldots . .}=[1,1,7,2,4,1,1,1,4,3,1,3,13,2] .
$$

$$
\begin{gathered}
3+\frac{1}{13+\frac{1}{2}} \\
H_{6}=2+\frac{1}{11+\ldots . .}=[2,11,52,2,1,1,1,3,2,8,1,5,2] .
\end{gathered}
$$

$$
1+\frac{1}{5+\frac{1}{2}}
$$

$$
H_{7}=2+\frac{1}{3+\ldots \ldots}=[2,3,10,2,11,1,1,2,4,1,7,2,2,2,1,2,5]
$$




$$
\begin{gathered}
H_{9}=2+\frac{1}{1+\ldots . .} \\
12+\frac{1}{3+\frac{1}{16}}
\end{gathered}=[2,1,6,1,1,2,1,6,1,3,1,1,1,4,1,3,1,2,1,3,12,3,16] .
$$

\section{CONCLUSION}

The continued fraction expansion algorithm has many specific features. However, the continued fraction expansion of any number or quotients of numbers is the efficient process of finding its best rational approximations. In fact, many continued fractions can be obtained in a similar way for different quotients with different powers.

\section{REFERENCES}

[1] Carlo Sanna, Michel Waldschmidt - Continued Fractions: Introduction and Applications, Proceedings of the Roman Number theory Association, vol.2, No.1, pp. 61 - 81, (2017).

[2] Pringsheim, A., Ueber die Convergenz unendlincher kettenbruche, Sitzungsber der math. Phys. Klasse der kgl. Bayer. Akad. Wiss., Munchen, 28, pp. 295-324, (1898).

[3] Muller, J.H.T., Lehrbuch der Mathematik, erster Teil die gesamte Arithmetik enthaltend, Halle, (1838).

[4] Rogers, L.J., On the representation of certain asymptotic series as convergent continued fractions. Proc. London Math. Soc. 2 (4), pp.72-89, (1907).

[5] Ramanujan, S., The lost note books and other unpublished papers. (Introduction by G.E. Andrews) Narosa Publishing House, New Delhi, pp. 44, (1988).

[6] Andrews, G.E., An Introduction to Ramanujan's 'Lost' Notebook. Amer. Math. Monthly 86, 89-108, (1979).

\section{AUTHOR'S BIOGRAPHY}

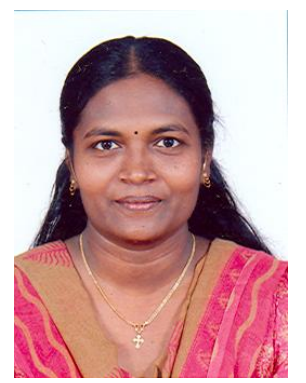

Dr. Roselin Antony is working as Associate Profesor (Mathematics) in Department of Mathematics, Mekelle University, Ethiopia. She has vast experience in the field of Number Theory and Special Functions. She has published many research papers and one Book entitled "Handbook of Mathematics Formulae (ISBN: 9788172338695)"

Citation: Roselin Antony, " Continued Fractions of Different Quotients ", International Journal of Scientific and Innovative Mathematical Research, vol. 5, no. 11, p. 20-31, 2017., http://dx.doi.org/10.20431/23473142.0511003

Copyright: (C) 2017 Authors. This is an open-access article distributed under the terms of the Creative Commons Attribution License, which permits unrestricted use, distribution, and reproduction in any medium, provided the original author and source are credited. 